Citation: Lastname, F.; Lastname, $\mathrm{F}^{22}$ Last-name, F. Title. Antibiotics 2021 10, x. https://doi.org/10.3390/xxxxx 24

\section{Received: date}

Accepted: date

Published: date

Publisher's Note: MDPI stays neu- 27 tral with regard to jurisdictional 28 claims in published maps and insti- 29 tutional affiliations.

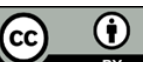

Copyright: (C) 2020 by the authors. 33 Submitted for possible open access 34 publication under the terms and 35 conditions of the Creative Common36 Attribution (CC BY) license $\quad 37$ (http://creativecommons.org/licenses8 /by/4.0/).

39
${ }^{1}$ Univ. Grenoble Alpes, CNRS, Grenoble INP, TIMC, Grenoble, France.

* Correspondence: hugomh@gmx.fr

\begin{abstract}
:
Failure of antibiotic therapies causes $>700,000$ deaths yearly and involves both bacterial resistance and persistence. Persistence results in the relapse of infections by producing a tiny fraction of pathogen survivors that stay dormant during antibiotic exposure. From an evolutionary perspective, persistence is either a 'bet-hedging strategy' that helps to cope with stochastically changing environments or an unavoidable minimal rate of 'cellular errors' that lock the cells in a low activity state. Here, we analyzed the evolution of persistence over 50,000 bacterial generations in a stable environment by improving a published method that estimates the number of persister cells based on the growth of the reviving population. Our results challenged our understanding of the factors underlying persistence evolution. In one case, we observed a substantial decrease in persistence proportion, suggesting that the naturally observed persistence level is not an unavoidable minimal rate of 'cellular errors'. However, although there was no obvious environmental stochasticity, in most cases the persistence level was maintained during 50,000 bacterial generations, and even increased in few cases.
\end{abstract}

Keywords: antibiotic persistence, evolution, Escherichia coli, beta-lactam, ampicillin, fluoroquinolones, ciprofloxacine, Start-Growth-Time, bacterial quantification.

\section{Introduction}

The current evolution of bacterial resistance to antibiotics brings humanity back to a situation reminiscent of the 'pre-antibiotic era' [1,2], mostly owing to the impressive bacterial adaptive properties. This alarming situation results in the death of more than 700,000 people every year. A worst-case scenario anticipates that this number might rise up to 10 million deaths per year by 2050, about three times the COVID-19 death rate [2]. In Europe in 2015, this caused 30,000 deaths per year, twice that in 2007 [3].

To cope with high antibiotic concentrations, bacteria rely on various processes that lead to different population dynamics [4-6]. These differences can be used to tentatively classify them. I) Resistance allows bacteria to grow under such antibiotic stress. II) Tolerance slows down the death rate of most of the population. III) Persistence involves only a tiny proportion of the population, from $10^{-6 \%}$ to $1 \%$ in natural populations [7], that benefits from a low death rate associated with low metabolic activity. This persistent state allows bacterial cells to cope with a broad range of stresses, albeit being genetically susceptible. Indeed, the size of a population exposed to antibiotics and containing persister cells first quickly decreases owing to the death of susceptible and metabolically-active cells, before experiencing a much slower decrease due to the death of persister quiescent cells. This physiological state is not heritable; hence, if a population regenerated from the persister sub-population is re-exposed to the antibiotic, a similar phenotypic heterogeneity 
will typically be observed again. This phenomenon allows growth rescue and restart after many stresses including nutrient depletion, temperature change, acid, oxidative or osmotic challenges, phage infection, and exposure to heavy metals or antibiotics [6,8].

Persistence is a natural process that evolved before the anthropogenic use and abuse of antibiotics. It might exist in all living organisms as it has been observed in all studied bacterial species [6,9], in eukaryotic cells resulting in cancer relapse [9-12], and even accidentally in digital organisms that learned to play dumb randomly at a low rate [13]. Understanding the evolutionary forces driving persistence emergence and maintenance may have far-reaching sanitary consequences. Indeed, persistence contributes to the relapse of many infections [14-17], including recurrence of mycobacterial infections in $10 \%$ of patients [18]. Moreover, persistence may have indirect effects on the evolution of antibiotic resistance as it can increase: i) mutation rates, ii) horizontal gene transfer rates, and iii) survival to antibiotics of susceptible cells by allowing repeated antibiotic exposures that select for progressive increase of antibiotic resistance $[6,19]$. Therefore, persistence is likely an important driver of antibiotic resistance evolution.

Although first described more than 70 years ago [20], the evolutionary forces driving persistence are still not well understood. It is important to emphasize that persistence is a typical case of phenotypic stochasticity, i.e., genetically identical bacterial cells that behave differently in given environments. This phenotypic stochasticity may either pre-exist to the stress (type I persister cells) or be induced by it (type II persister cells) $[15,21]$. Beyond persistence, stress-induced phenotypic stochasticity is a general feature of living organisms that is observed in many taxa and organization levels, such as increased developmental noise in plants [22], bears [23], fishes [24], Drosophila [25] (for reviews, see [2630]).

There is a current debate about the evolutionary meaning of phenotypic stochasticity [31-34], including persistence. It is hypothesized to be either an unavoidable consequence of biological constraints or an adaptive process related to bet-hedging. According to the first hypothesis, any phenotype has a minimal amount of random variance. In addition, this variance can be increased by stresses which may lead to random abnormal phenotypes by causing cell alterations. Indeed, stresses will hamper mechanisms selected to reduce phenotypic stochasticity (developmental stability [35]), thereby resulting in a minimal amount of phenotypic stochasticity. For example, acid and temperature stresses can lower protein conformational stability [36]. In the second hypothesis, by increasing the probability that few individual cells are adapted to the stress, this variability may be advantageous and thus selected for, leading to the evolution of bet-hedging [37]. For example, cells from all life kingdoms have a mistranslation mechanism that is activated as a stress response [30]. This discrepancy between the two hypotheses is particularly relevant for persistence [7]. Levin et al. [38] emphasized that persistence might be related to errors in cellular processes that block cells in almost inactive states. In agreement, a large number of mutations can result in increased persistence frequency [39], while no mutation has ever been found to prevent it [40], supporting the hypothesis that persistence is merely a result of biological constraints. On the other hand, some persister cells can actively pump antibiotics out from cells [41,42], and their proportion in the population may evolve rapidly, suggesting an adaptive process. Indeed, chronic bacterial infections evolved an increased rate of persistence after antibiotic treatment [43-46].

Laboratory evolution experiments confirmed the high persistence evolvability. In the presence of antibiotics, a 100- to 1000-fold increase in persistence has indeed been reported after only two to three cycles of antibiotic selection (less than five days) [47]. In contrast to antibiotic resistance, the production of a small proportion of persister cells entails low cost to the population. Therefore, restoring the initial level of persistence is a slow process, requiring hundreds to thousands of generations [47]. Hence, long-term experimental evolution in the absence of killing stressors such as antibiotics is particularly relevant to study persistence evolution. Here, we used the long-term evolution experiment (LTEE) that was 
initiated in 1988, during which twelve populations are propagated from a common Escherichia coli ancestor in a glucose-limited, antibiotic-free environment [48]. We recently showed that susceptibility to many antibiotics increased over time in these conditions in which bacteria were selected for faster growth for more than three decades [49].

We investigated the evolution of persistence for ampicillin and ciprofloxacin by comparing their effects in the ancestor and evolved clones sampled in each of the twelve populations up to 50,000 generations. We improved a high-throughput methodology [50] to estimate the prevalence of persisters while accounting for growth rate heterogeneity.

\section{Results}

We performed two sets of analyses. First, in the 'LTEE-50K' analysis, we compared persistence to the two antibiotics ampicillin and ciprofloxacin of one clone sampled at 50,000 generations from each of the 12 populations of the LTEE to their respective ancestors REL606 and REL607. Second, in the 'Ara-2_S_L' analysis, we investigated the persistence level within the population called Ara-2 (Table 1) in which an adaptive diversification event occurred. Indeed, two phenotypically-distinct ecotypes, called S and L, emerged by generation 6,500 and co-exist ever since [51]. We sampled 10 evolved clones from Ara-2, including one from each generation 2,000 and 5,000 before the emergence of this polymorphism, and one $S$ and $L$ clone from each generation 6,500, 11,000, 20,000 and 50,000 (Table 1).

Table 1: List of theLTEE-derived clones used in this study.

\begin{tabular}{|c|c|c|c|c|c|}
\hline Clone & LTEE population & Generation & Mutator state* & \multicolumn{2}{|c|}{ Analyses** } \\
\hline 606 & Ancestor (Ara-) & 0 & $\mathrm{~N}$ & LTEE-50K & Ara-2_S_L \\
\hline 607 & Ancestor (Ara +$)$ & 0 & $\mathrm{~N}$ & LTEE-50K & Ara-2_S_L \\
\hline 11330 & Ara-1 & 50,000 & $\mathrm{M}$ & LTEE-50K & \\
\hline $1165 \mathrm{~A}$ & Ara-2 $\left(\mathrm{BC}^{* * *}\right)$ & 2,000 & $\mathrm{~N}$ & & Ara-2_S_L \\
\hline $2180 \mathrm{~A}$ & Ara-2 $\left(\mathrm{BC}^{* * *}\right)$ & 5,000 & M & & Ara-2_S_L \\
\hline $6.5 \mathrm{KS} 1$ & Ara-2 (S) & 6,500 & $\mathrm{M}$ & & Ara-2_S_L \\
\hline $6.5 \mathrm{KL} 4$ & Ara-2 (L) & 6,500 & M & & Ara-2_S_L \\
\hline 11KS1 & Ara-2 (S) & 11,000 & $\mathrm{M}$ & & Ara-2_S_L \\
\hline 11KL1 & Ara-2 (L) & 11,000 & $\mathrm{M}$ & & Ara-2_S_L \\
\hline 20KS1 & Ara-2 (S) & 20,000 & $\mathrm{M}$ & & Ara-2_S_L \\
\hline 20KL1 & Ara-2 (L) & 20,000 & $\mathrm{M}$ & & Ara-2_S_L \\
\hline 13335 & Ara-2 (S) & 50,000 & $\mathrm{~N}$ & LTEE-50K & Ara-2_S_L \\
\hline 11333 & Ara-2 (L) & 50,000 & M & LTEE-50K & Ara-2_S_L \\
\hline 11364 & Ara-3 & 50,000 & $\mathrm{M}$ & LTEE-50K & \\
\hline 11336 & Ara -4 & 50,000 & $\mathrm{M}$ & LTEE-50K & \\
\hline 11339 & Ara-5 & 50,000 & $\mathrm{~N}$ & LTEE-50K & \\
\hline 11389 & Ara-6 & 50,000 & $\mathrm{~N}$ & LTEE-50K & \\
\hline 11392 & $\mathrm{Ara}+1$ & 50,000 & $\mathrm{~N}$ & LTEE-50K & \\
\hline 11342 & Ara +2 & 50,000 & $\mathrm{~N}$ & LTEE-50K & \\
\hline 11345 & $\mathrm{Ara}+3$ & 50,000 & $\mathrm{M}$ & LTEE-50K & \\
\hline 11348 & Ara +4 & 50,000 & $\mathrm{~N}$ & LTEE-50K & \\
\hline 11367 & Ara +5 & 50,000 & $\mathrm{~N}$ & LTEE-50K & \\
\hline 11370 & Ara+6 & 50,000 & $\mathrm{M}$ & LTEE-50K & \\
\hline
\end{tabular}

*The mutator $(\mathrm{M})$ or non-mutator $(\mathrm{N})$ state is indicated.

"See text below, section "Rationale of data analyses".

${ }^{* * *} \mathrm{BC}$, before co-existence. 
To quantify persistence after a 5-hour antibiotic exposure, we performed a ten-fold dilution cascade, from $10^{0}$ to $10^{7}$. For each dilution, we recorded the bacterial culture revival, i.e. the presence/absence of growth after $24 \mathrm{~h}$ and, if revival, we quantified the number of persister cells by analyzing the growth curve (initial OD approach described hereafter). Absence of growth after $24 \mathrm{~h}$ was related to either the absence of persister cells or a number of persister cells too low for their revival and detection. In this case, we set the number of persister cells to zero.

\subsection{Validation of the initial OD approach to quantify persister cells}

We adapted the approach used by Hazan et al. [50] by recording the intercept of a linear model fitted to the $\log _{2}$ of the growth curve, which is an estimate of the initial OD (hereafter, initial OD), instead of recording the time needed to reach a threshold OD called the Start Growth Time (SGT). This approach avoids biases induced by growth rate variations and can detect tiny variations in initial OD (Appendix A). However, as the SGT approach [50], it assumes that the lag time for cell regrowth is unaffected by the antibiotic treatment, although it is known to differ between persister and non-persister cells [21]. We accounted for this approximation by referring, for this estimated number of persister cells (CFU number), to an Equivalent Number of Normal Cells (\#EqNC), i.e., the initial number of cells that would have yielded the same initial OD in the absence of antibiotic exposure.

To validate this initial OD approach, we checked that, on average, the ten-fold dilution series yielded ten-fold differences in the \#EqNC. In that case, the average of the slopes of models predicting $\log _{10}(\# E q N C)$ by $\log _{10}$ (dilution) should be equal to one. We found an average of 0.97 with a bootstrap $95 \% \mathrm{CI}$ of [0.90; 1.05].

\subsection{Evolution of persistence in the 'LTEE-50K' analysis}

For each growth curve, we estimated initial OD and used standard curves specific to each strain for conversion into CFUs (\#EqNC, Appendix A). We used a linear mixed model to estimate the mean \#EqNC and its confidence interval for each clone (Table 1) and treatment (ampicillin, ciprofloxacin, no antibiotics). This model predicts the $\log _{2}$ (\#qNC) of each growth curve as a function of, as fixed effects, $\log _{10}$ of the dilution, antibiotic treatment, clone ID, and two second-order interactions with the antibiotic treatment, and random effects on these fixed effects. All fixed effects were highly significant (Table 2).

Table 2: Tests of the fixed effects of the model analyzing the \#EqNC.

\begin{tabular}{llll}
\hline Variable & $\mathrm{df}$ & $F$-value & $p$-value \\
\hline $\log _{10}($ dilution$)$ & $1,100.58$ & 595.11 & $<0.001$ \\
Clone ID & $23,59.92$ & 48.86 & $<0.001$ \\
Antibiotic & $2,1342.65$ & 144.94 & $<0.001$ \\
Antibiotic $\times \log _{10}($ dilution$)$ & $2,131.47$ & 7.63 & $<0.001$ \\
Antibiotic $\times$ clone ID & $44,399.89$ & 9.45 & $<0.001$ \\
\hline
\end{tabular}

P-values of fixed effects are based on F-tests with Satterthwaite's approximation. The corresponding numerator and denominator degrees of freedom (df) and statistics of the tests (F-values) are given.

2.2.1 Overall trends in the persistence level to ampicillin and ciprofloxacin.

We analyzed the estimated \#EqNC for each investigated clone and treatment (coefficients of the model summarized in Table 2 and shown Figure 1). We found that the overall level of persistence to ciprofloxacin and ampicillin was similar (bootstrapped paired $t$-test implemented in the R package MKinfer: $p$-value $=0.22$ ), and positively correlated (Spearman rank correlation $=0.508 ; p$-value $=0.014$; Figure 1 ). However, this pattern varied according to the clones. Six of the twelve populations evolved a mutator phenotype owing to mutations in DNA repair genes before 50,000 generations (Table 2; [52]). We found no 
association between the persistence level and the mutator/non-mutator state of the populations (linear mixed model, $p$-value of the interaction between mutator state and treatment $=0.94$ ). Nevertheless, the positive correlation between persistence to ciprofloxacin and ampicillin was mostly driven by the non-mutator clones (Spearman rank correlations $=0.87$ and $0.08 ; p$-values $=0.003$ and 0.78 , respectively for the non-mutator and mutator clones). A permutation test comparing these two correlations yielded a p-value of 0.037 (50,000 permutations of $\left.\left|\sigma_{\text {mutator }}-\sigma_{\text {non-mutator }}\right|\right)$.

The among-strain variability for persistence to ciprofloxacin was higher than to ampicillin (Ansari-Bradley test $\mathrm{AB}=331 ; p$-value $=0.016$; Figure 1 ). There was no correlation between persistence and resistance to these antibiotics [49] (Spearman rank correlations = 0.365 and $0.074 ; p$-values $=0.22$ and 0.81 for ampicillin and ciprofloxacin, respectively).

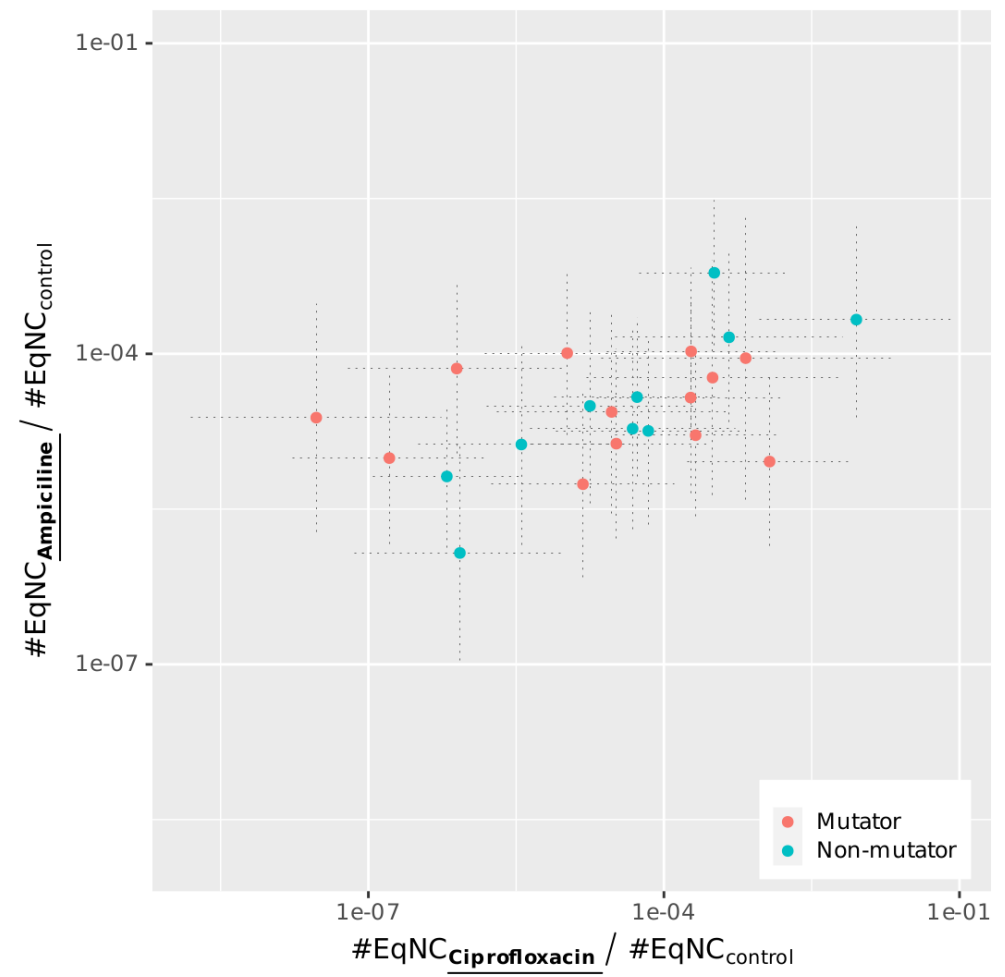

Figure 1: Persistence to ampicillin vs. ciprofloxacin in evolved clones sampled from each of the 12 LTEE populations.

For each clone (Table 2), the abundance of persister cells to ampicillin and ciprofloxacine was quantified by the ratio between the \#EqNC in the treatment and the control. Each dot corresponds to a given clone and gives the abundance of persister cells to the two antibiotics. Dotted lines give the $95 \%$ CI. These values were obtained from the coefficients of the models (Table 2).

\subsubsection{Evolution of persistence after 50,000 generations of evolution}

We first showed that there was no significant difference in the level of persistence between the two ancestral clones REL606 and REL607 ( $p$-values $=0.20$ and 1 for ampicillin and ciprofloxacin, respectively). Then, we performed both comparisons of each evolved clone to each of the two ancestral strains and pairwise comparisons among the 13 clones sampled at generation 50,000 using the model coefficients (Table 2; Figure 2). Evolution of persistence to ciprofloxacin of the $S$ clone from population Ara-2 was the only significant difference compared to the ancestors $(p$-value $=0.01$; Figure 2$)$. However, the pairwise 
comparisons among clones from generation 50,000 had more statistical power and detected significant changes in the level of persistence to ciprofloxacin. Persistence was: i/ higher in the Ara+4 clone than in clones from populations Ara-3, Ara-4, Ara-5, Ara-6, Ara+2, Ara +3 and the $S$ clone from Ara-2; ii/ higher in the Ara 5 and Ara+6 clones than in clones from populations Ara-4 and Ara+2 and the S clone from Ara-2 (Supplementary materials, Table S1, S2, and S3).

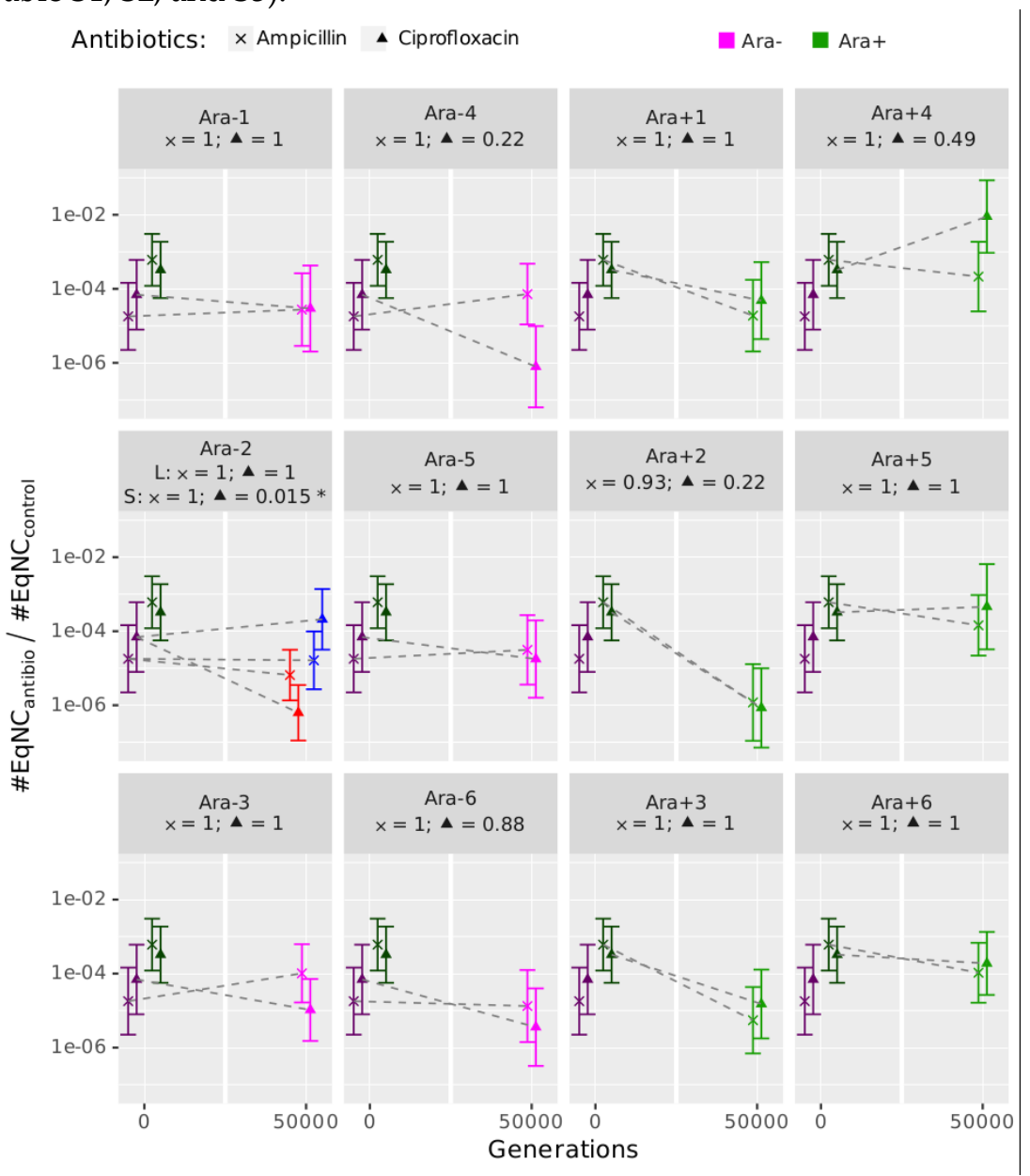

Figure 2: Evolution of persistence to ampicillin $(x)$ and ciprofloxacin $(\boldsymbol{\Delta})$ in evolved clones sampled from the 12 LTEE populations. For each antibiotic, we compared the level of persistence of each evolved clone sampled at generation 50,000 to the one in each of the two ancestors REL606 and REL607, and to be conservative, only the least significant of the two comparisons was kept for each evolved clone. The p-values for each antibiotic are shown below the name of the population (and for each of the $S$ and L ecotypes in population Ara-2, in red and blue respectively). These values were obtained from the coefficients of the models summarized in Table 2. 95\% confidence intervals are shown. Dark symbols represent the ancestor strains, pink the clones from the Ara-1 to Ara-6 populations, and green the clones from the Ara+1 to Ara+6 populations.

Significance codes: 0 ***' $0.001^{* * \prime} 0.01^{* *} 0.05$ '. 0.1 ' ' 1 .

\subsection{Evolution of persistence in the 'Ara-2_S_L' analysis}

We investigated the interplay between the adaptive diversification event that occurred in population Ara-2 and the evolution of persistence. The emergence of diversification was detected between generations 5,000 and 6,500, leading to the co-existence of two ecotypes called Ara-2L and Ara-2S [51]. From generation 11,000 to 50,000, each Ara$2 \mathrm{~S}$ sampled clone had a significantly lower level of persistence to ciprofloxacin compared 
to both the ancestors and the corresponding contemporary Ara-2L sampled clone, while there were no significant differences for persistence to ampicillin (Figure 3).

\section{A Ampicillin}

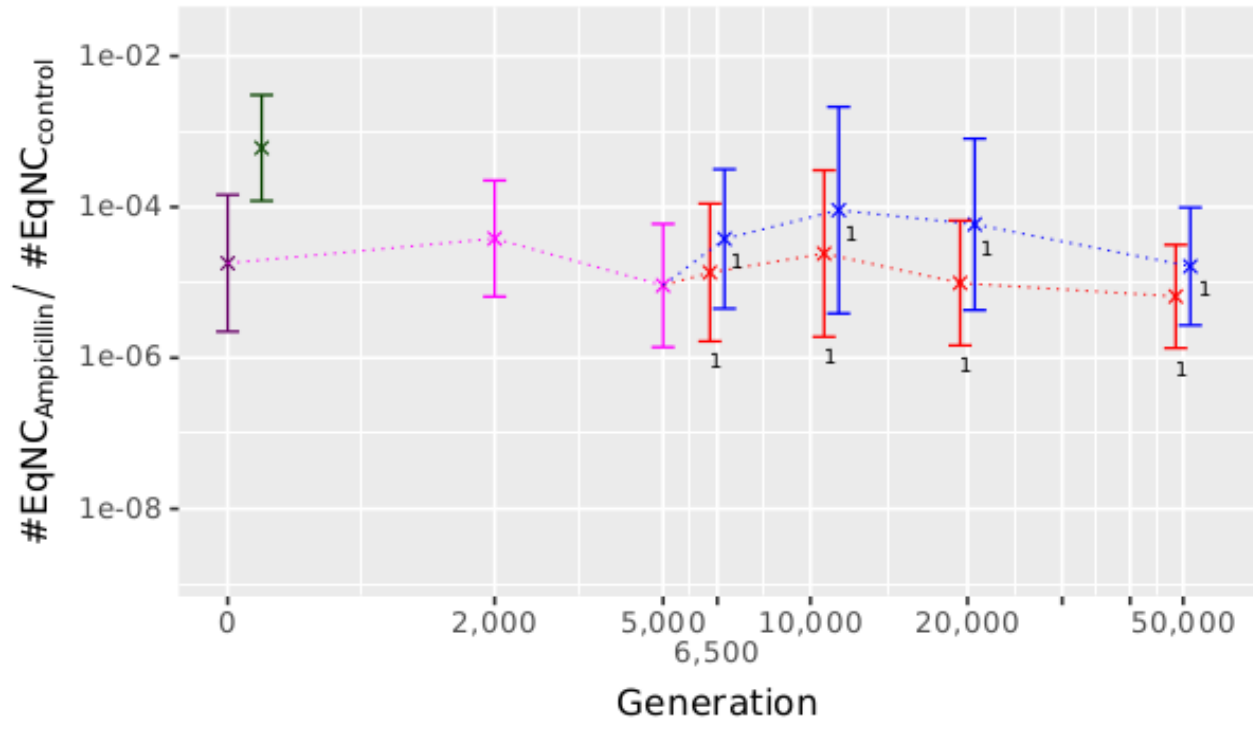

\section{B Ciprofloxacin}

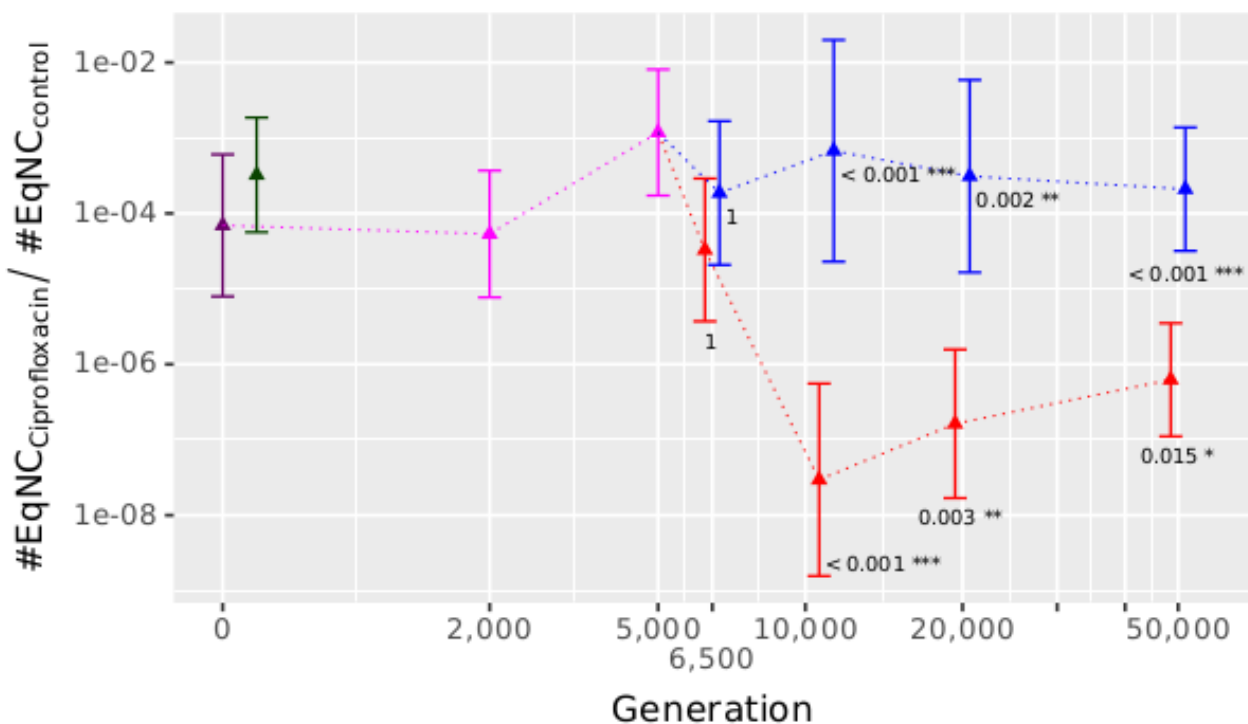

Figure 3: Evolution of persistence to ampicillin (A) and ciprofloxacin (B) in evolved clones sampled from the Ara-2 population. Dark symbols represent the ancestor strains REL606 and REL607, pink the Ara-2 evolved clones sampled before the adaptive diversification event, red and blue the evolved clones from the $S$ and $L$ ecotypes, respectively. P-values close to the Ara-2S evolved clones refer to the comparisons to the ancestors, and $\mathrm{p}$-values close to the Ara-2L evolved clones refer to the comparison between the co-existing contemporary $S$ and L evolved clones. 95\% confidence intervals are shown.

\section{Discussion}

We investigated the evolution of bacterial persistence during the LTEE over 50,000 generations, corresponding to 22 years during which bacterial cells were maintained in a 
defined antibiotic-free environment [53]. Environmental variations included the daily cycles of feast and famine and changes produced by bacteria themselves, as for example secretion of metabolic byproducts [54]. We improved a high-throughput method to quantify persister cells by accounting for growth rate heterogeneity. Despite evolutionary potential for lower persistence, it was not observed in most cases in this antibiotic-free environment.

Studying persistence is complex because: $i$ / persistent cells are genetically identical to non-persistent cells, ii/ it is rare in entire populations, iii/ persistence state is not heritable, and iv/ once in the persistence state, cells do not multiply. Here, we improved a previously published method [50] to quantify persister cells. This approach relied on analyzing the growth curve of the bacterial population that recovered from the stress that killed non-persistent cells. The more persister cells, the faster the growth of the reviving population, which thereby becomes detectable. This approach assumed that the growth rate is constant among treatments. We however showed that this was not the case for persister cells (Supplementary material, Fig. S1). Therefore, we fitted linear models on the $\log _{2}$ of each growth curve, which estimates the growth rate and initial population density.

We detected no relationship between the levels of persistence and antibiotic resistance [49], thereby showing that the two phenomena are related to two different pathways in the conditions of the LTEE where there is no antibiotic pressure but selection for growth. Similar results were observed in natural strains of Pseudomonas spp. [55].

Overall, among the LTEE clones, the level of persistence to the two antibiotics was similar and positively correlated, suggesting similar physiological pathways. However, the positive correlation was only driven by non-mutator strains, and the among-strain variation was significantly higher for ciprofloxacin than ampicillin. In addition, we detected no significant differences among clones for ampicillin by contrast to ciprofloxacin. This suggests the existence of different types of persister cells, some being shared between ampicillin and ciprofloxacin, and some that are specific to each antibiotic. The observed among-clone variation would result from both types of persister cells, those common to the two antibiotics explaining the correlation in the non-mutators and those specific to each antibiotic explaining the difference between the two antibiotics in among-clone variance. In agreement with this diversity of persister cells, it has been observed that a large panel of pathways can trigger persistence [15], and the correlation between persistence to different antibiotics is variable. Hence, a marginally significant correlation between the persistence to ampicillin and nalidixic acid was found in environmental samples of $E$. coli [56], but no correlation between the persistence to these two antibiotics and to ciprofloxacin, albeit ciprofloxacin and nalidixic acid share a similar mechanism of action. A similar analysis by Stewart and Rozen [57] revealed no correlation in the level of persistence to ampicillin, streptomycin, and norfloxacin.

Persistence in the ancestral strains REL606 and REL607 may originate from either past selection by environmental stochasticity or minimal amount of 'cellular errors'. In the former case, we would expect no evolution of persistence and a higher amount of persister cells in mutator clones because the mutational target for higher persistence is large [39]. In addition, if persistence in the ancestor results from adaptation to former environmental stochasticity, the naïve prediction would be a reduced persistence level in most, if not all, LTEE populations that evolved under strong selection for improved growth during 22 years in a constant environment. Indeed, bacteria have a strong capacity to adapt to cyclic or correlated environmental changes [58-61], and shifting to a random dormancy state should be costly as any growing mutant among other dormant cells would have a higher growth rate.

By contrast to these two alternative predictions, we detected no relationship between persistence and mutator state, and only one of the 13 analyzed clones (from the Ara-2S ecotype) showed significant evolution toward lower persistence. Evolved clones from the two populations Ara-4 and Ara +2 evolved lower persistence that was only significant when compared to clones that evolved higher persistence (from populations Ara +4 , Ara +5 
and Ara+6). These results show an evolutionary potential for both higher and lower persistence, but in most cases, the stable LTEE environment did not select for low persistence.

The only clone revealing lower persistence is the 50,000-generation Ara-2S clone. Interestingly, its relative fitness compared to its contemporary clone from the Ara-2L ecotype is higher during stationary phase than exponential phase [62,63]. Indeed, while the Ara-2L clone was starving from glucose, the Ara-2S clone consumed the acetate produced during growth on glucose [64]. Hence, it might favor dormancy of the Ara-2L clone during stationary phase to lower both its energy consumption and death rate, while the Ara$2 \mathrm{~S}$ clone was actively growing on acetate. This hypothesis is particularly appealing since starvation has been shown to be a main natural cause of persistence evolution $[6,21,65]$. Hence, persistence may provide benefits to starvation in natural environments because feast and famine phases are poorly predictable. By contrast in the LTEE, bacteria experience a seasonal and predictable environment every day since more than three decades, oscillating between exponential phase (feast) and stationary phase (famine). Because of such reduced stochasticity in the LTEE between the different growth seasons, randomly switching a small proportion of cells into dormancy may not be a beneficial strategy.

Further studies may investigate the influence of the daily starvation phase in the maintenance of persistence during the LTEE. Alternatively, the observed variability among the evolutionary trajectories of persistence might result from pleiotropic interactions between persistence and other selected traits. According to this scenario, the variability in evolutionary trajectories of different populations would result from some contingencies and would be a side effect of the divergence of populations. Understanding these pleiotropic interactions would be very useful to optimize the treatments of infections or cancers. Indeed, this might allow to pre-evolve an infecting bacterial cell population or a cancer cell population to have a low persistence to the future treatment.

The conundrum of the unexpected maintenance of persistence in a stable environment over 50,000 bacterial generations, albeit evolutionary potential for decreasing the amount of persistence as observed in the Ara- $2 S$ clone, highlights the LTEE importance for challenging and testing our understanding of evolution.

\section{Materials and Methods}

\section{Strains}

We used a total of 23 E. coli clones that are all derived from the LTEE: the two ancestral clones REL606 and REL607, the latter being a spontaneous Ara+ revertant of REL606 $[53,66]$, one evolved clone sampled at 50,000 generations from 11 of the 12 LTEE populations, and 10 evolved clones from the population called Ara-2 (Table 1). Indeed, the population Ara-2 experienced an adaptive diversification event during which two phenotypically-distinct ecotypes, called S and L, emerged by generation 6,500 and co-exist ever since [51]. Therefore, we sampled one Ara-2 evolved clone from generations 2,000 and 5,000 before the emergence of this polymorphism, and one $\mathrm{S}$ and $\mathrm{L}$ clone at each of the generations 6,500, 11,000, 20,000 and 50,000 (Table 1).

\section{Measuring the proportion of persister cells}

For each LTEE-derived clone, we estimated the amount of persister cells after exposure to each of two bactericidal antibiotics belonging to two different families, ampicillin and ciprofloxacin, by hypothesizing that the larger the persister population is, the faster regrowth will happen and therefore be detectable by optical density [49, see below].

All clones were grown in DM1000 medium (Davis Minimal broth supplemented with glucose at $1 \mathrm{~g} / \mathrm{L}$ ) at $37^{\circ} \mathrm{C}$ and $180 \mathrm{rpm}$. This high glucose concentration improves the accuracy of the OD measure during regrowth after antibiotic exposure. After overnight culture, stationary-phase cells were diluted at 1/1000 in $500 \mathrm{~mL}$ DM1000. After 2.5h of growth (mid-exponential phase), we divided each culture into nine 5-mL tubes that were subdivided into three groups, i.e., three technical replicates per group. In the two first groups, 
we added either ampicillin or ciprofloxacin at a final concentration of 100 and $1 \mu \mathrm{g} / \mathrm{mL}$, respectively. The third group was used as an antibiotic-free control. After 5 hours at $37^{\circ} \mathrm{C}$ and $180 \mathrm{rpm}$, we removed antibiotics by three successive washes consisting in 5-min centrifugation at $1500 \mathrm{~g}$, removal of $90 \%$ of the supernatant and resuspension of the pellet in antibiotic-free DM1000. Finally, cells were resuspended in $200 \mu \mathrm{L}$ DM1000 and each tube content was transferred into a well of a 96-well microplate (Thermo Scientific 260860). We performed for each initial tube tenfold dilution cascades from $10^{0}$ to $10^{7}$ fold, and monitored growth in an Infinite M200 microplate reader (Tecan $\left.{ }^{\circledR}\right)$ to quantify the proportion of persister cells for each clone. We recorded the OD 600 every $15 \mathrm{~min}$ for $24 \mathrm{~h}$ that we will use as the 'time' variable in our analysis (see below). In addition for each antibiotic-free control tube, we estimated the CFU number to compute the relationship between the number of cells and the time after which regrowth was detected by OD. We performed at least three biological replicates for each clone.

\section{Rationale of data analyses}

All analyses were performed to: i) compare the persistence frequency in each of the 12 evolved clones sampled at 50,000 generations to the corresponding ancestor REL606 or REL607, and ii) analyze the evolutionary dynamics of persistence in the population Ara2 in which the $S$ and L ecotypes emerged by generation 6,500 and co-exist since then [67]. We refer hereafter to these two analyses as LTEE-50K and Ara-2_S_L, respectively (Table $1)$.

\section{Quantification of the population size of persister cells}

We improved a previous approach [50] that is similar to qPCR as the time needed to detect an increase in the signal (here, $\mathrm{OD}_{600}$ ) is proportional to the initial amount of material (here, the number of persisters cells). Hence, the time needed to reach a given $\mathrm{OD}_{600}$ threshold is defined as the Start Growth Time, SGT [50]. This approach however assumed that persister cells have both a growth rate and a lag phase that are similar to the cells of the cultures used for the standard curve, albeit they were not exposed to antibiotics (Fig. A1e). Comparing the growth rates of each strain in each treatment showed that persister cells actually had a slower growth rate than other cells. For unknown reasons, this differential effect was stronger for ciprofloxacin than ampicillin (Supplementary material, Fig. S1). Therefore, we developed an alternative approach based on a statistical model that predicted the $\log _{2}$ of the OD600 observed during exponential growth as a function of time (Supplementary material, Heuristic selection of the exponential growth phase). These models estimate both the growth rate (slope) and initial OD600 (intercept; hereafter, initial (D) for each growth curve. This approach can detect tiny variations in initial OD as they increased during exponential growth (for more details, see Appendix A). Using this approach, we obtained initial OD for each growth curve and converted it into cell numbers using standard curves obtained for each strain by quantifying both initial OD and CFUs in dilution series (Fig. A1d).

However, while this approach accounted for growth rate heterogeneity, it still assumed that the mean lag time for cell regrowth was identical in all strains and treatments. Hence, we refer to this estimated number of persister cells (number of CFU) as an Equivalent Number of Normal Cells (\#EqNC), corresponding to the initial number of cells that, in the absence of antibiotics, would have produced the same initial OD. In the absence of growth after $24 \mathrm{~h}$, the \#EqNC was set to zero. To validate this initial OD approach, we checked that the ten-fold dilution series indeed yielded ten-fold differences in the estimated amount of persister cells (\#EqNC). Specifically, we used the initial OD approach to estimate \#EqNC separately for each growth curve. Then, for each dilution series, we fitted a model predicting $\log _{10}(\# E q N C)$ by $\log _{10}$ (dilution). If the ten-fold dilutions resulted in an averaged ten-fold difference in the number of persister cells, these models should have a slope of one that we checked by bootstrapping the slopes. 


\section{Estimating and comparing persistence}

We estimated the level of persistence to ampicillin and ciprofloxacin in each strain by fitting a linear mixed model predicting $\log _{2}(\# \mathrm{EqNC})$ with as i/ fixed effects: the $\log _{10}$ of the dilution factor, the antibiotic treatment, the clone, and the two second-order interactions with antibiotic treatments, ii/ random effects: 'replicates' on the intercept, the three fixed effects 'dilution', 'treatment' and their interaction. The intercept of these models estimates the mean $\log _{2}(\# \mathrm{EqNC})$ in the non-diluted sample, i.e., when $\log _{10}$ of the dilution is equal to zero.

We fitted this model with the lme4 package [68] of the $\mathrm{R}$ version 4.0.3 and tested significance of effects with $\mathrm{F}$ tests and the Satterthwaite approximation for degrees-offreedom [69,70] (Table 2). We compared clones to each other using the $\mathrm{R}$ package 'multcomp' (version 1.4-17; [71]). For each antibiotic, we compared each evolved strain to the two ancestors REL606 and REL607. This first set of tests was used to: i/ check for the absence of significant differences between the two ancestors, and ii/ assess whether the evolved clones were significantly different from their ancestors. To be conservative when detecting changes, only clones that were significantly different from both ancestors were considered has being significantly different. We also performed pairwise comparisons between 50,000-generation clones to detect groups of clones that evolved in opposite directions. Finally, we used this test to compare contemporary co-existing clones Ara-2 L and $S$.

\section{Relationship between persistence and mutator phenotype}

To test for an effect of the mutator phenotype on persistence, we re-fitted the linear mixed model predicting $\log _{2}(\# \mathrm{EqNC})$ by setting the two variables clone and interaction between clone and treatment as a random instead fixed effect, and by adding, as fixed effect, the mutator state and its interaction with the treatment. Furthermore, we assessed the effect of the mutator state on the correlation between persistence to ampicillin and ciprofloxacin using Spearman rank correlations. Specifically, we measured and tested correlation $(\sigma)$ among all clones and then separately among mutator and non-mutator clones. We tested for the significance of the difference between mutator and non-mutator clones using 50,000 permutations of the statistic $\left|\sigma_{\text {mutator }}-\sigma_{\text {non-mutator }}\right|$.

Supplementary Materials: The following is available online at www.mdpi.com/xxx/s1, Table S1: 'Pairwise comparisons of the abundance of ciprofloxacin persister cells in the evolved clones sampled from each of the 12 LTEE populations at 50,000 generations'; Table S2: 'Pairwise comparisons of the abundance of ampicilin persister cells in the evolved clones sampled from each of the 12 LTEE populations at 50,000 generations'; Table S3: 'Pairwise comparisons of the abundance of ciprofloxacin persister cells in the evolved clones sampled from each of the 12 LTEE populations at 50,000 generations'; Figure S1: 'Effect of antibiotics on growth rates'; Supplementary method 1: 'Heuristic selection of the exponential growth phase'; Figure S2: 'Variability in background OD for the wells of a representative microplate'; Figure S3: 'Illustration of the detection of the exponential phase in the growth curve'

Author Contributions: Experimental design, D.S., J.G. and M.M.; Realization of the experiments, M.M., J.G., R.A. and H.M.H.; Design of the data analysis, H.M.H.; Data analysis, R.A., and H.M.H.; writing - original draft preparation, H.M.H; writing - review and editing, D.S., J.G., and H.M.H.; funding acquisition, D. S. All authors have read and agreed to the published version of the manuscript.

Funding: This research was funded by Agence Nationale de la Recherche, grant number ANR-18CE35-0005-04; by the Centre National de la Recherche Scientifique, IRP PredEvo; and by the Université Grenoble Alpes.

Institutional Review Board Statement: Not applicable.

Informed Consent Statement: Not applicable. 
Data Availability Statement: The data presented in this study are openly available in FigShare at doi: 10.6084/m9.figshare.19209690, reference number XXX Data are not yet publically available, but they can be accessed here : https://figshare.com/s/9782ddbebfc25e0bce4b .

Acknowledgments: We kindly thank Richard Lenski (Michigan State University, BEACON Center, USA) for the gift of strains and a long lasting collaboration. We thank Thomas Hindré for helpful discussions.

Conflicts of Interest: The authors declare no conflict of interest.

\section{Appendix A: Estimation of the Equivalent Number of Normal Cells (\#EqNC)}

We computed \#EqNC by comparing how fast growth was detected compared to a standard curve that was obtained by quantifying living cells within a sample unexposed to antibiotics through CFU counting. To quantify persister cells from growth curves, Hazan et al. [50] measured how fast growth was detected as the time OD reached a given threshold (Start Growing Time), as in GPCR analyses. It assumed however that all compared strains had similar growth rates which was not the case since they are affected by antibiotics (Supplementary material, Fig. S1). We therefore developed an alternative approach independent from this assumption. For each growth curve, we fitted a linear model to the $\log _{2}$ of the OD from which the background OD has been removed. The slope and the intercept of these models estimate the growth rate and $\log _{2}$ of the initial OD minus the background OD, respectively. The estimated initial OD is proportional to the number of initial cells and is unaffected by the growth rate. 
a

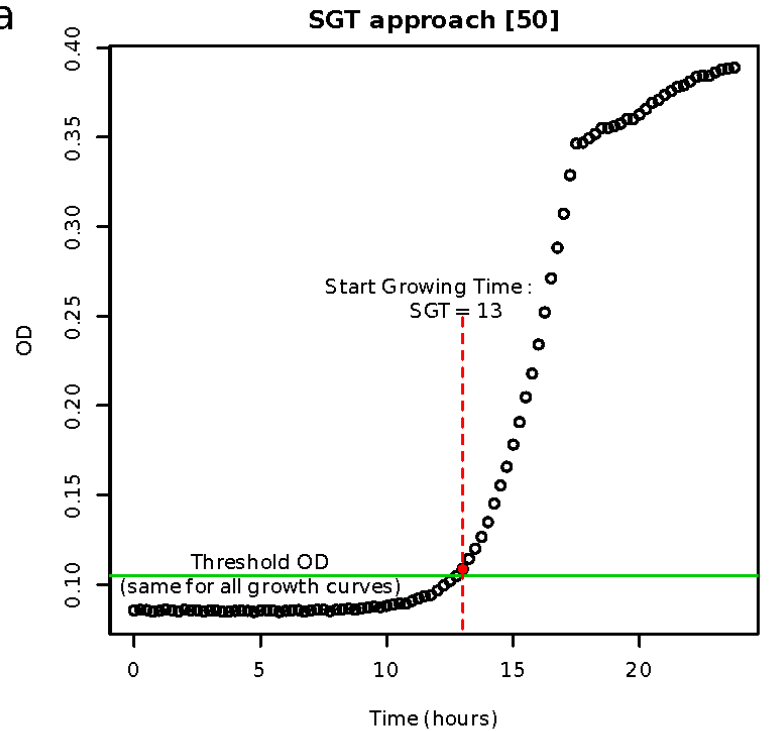

C

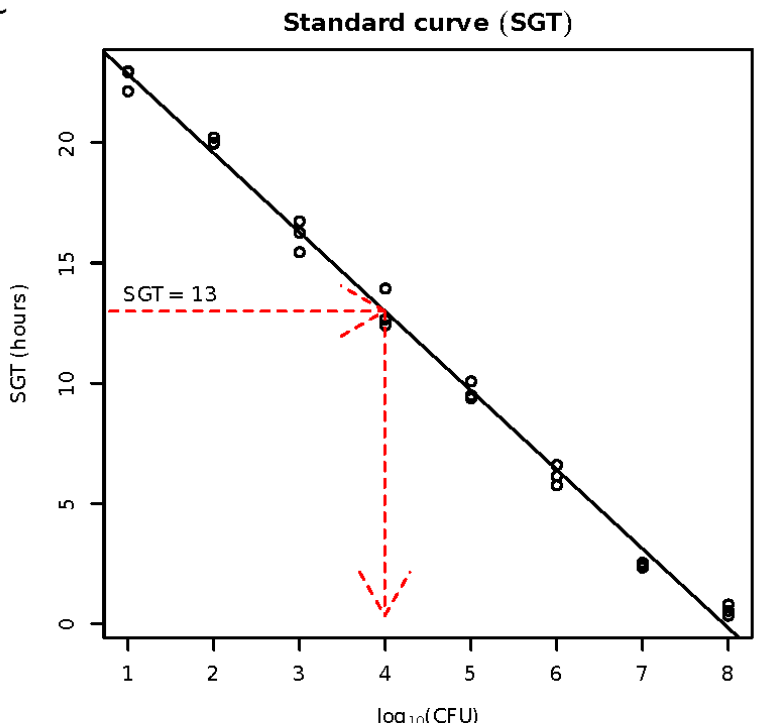

e

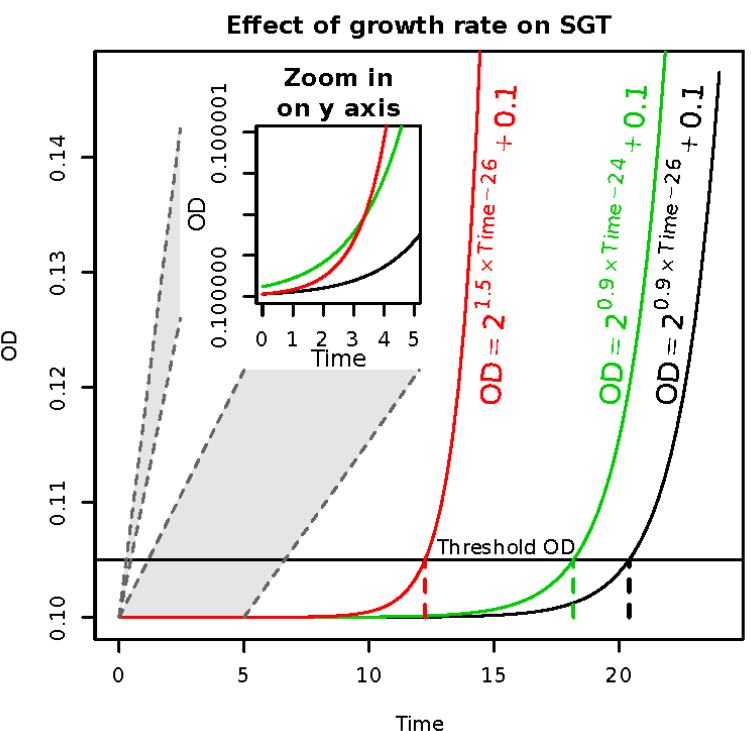

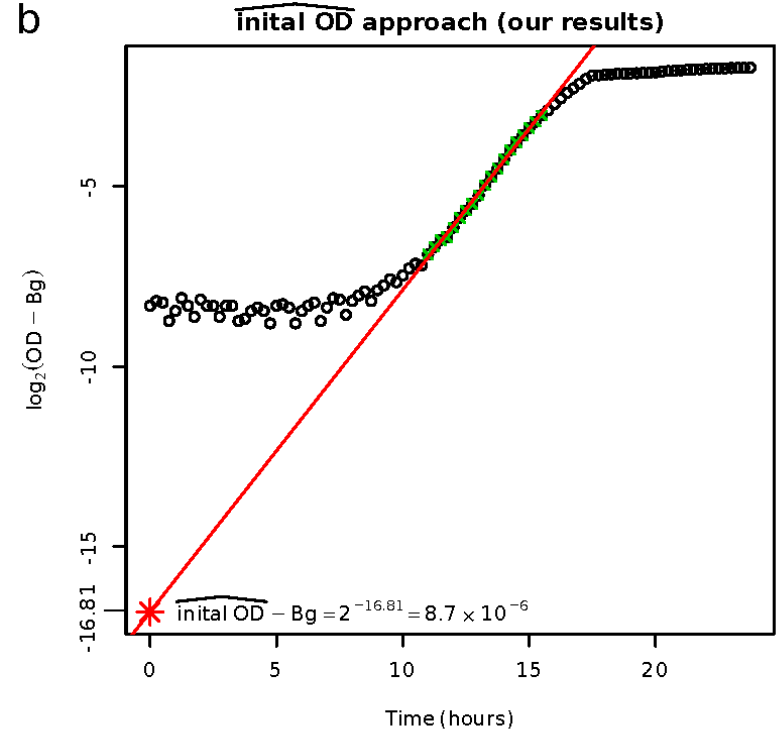

d
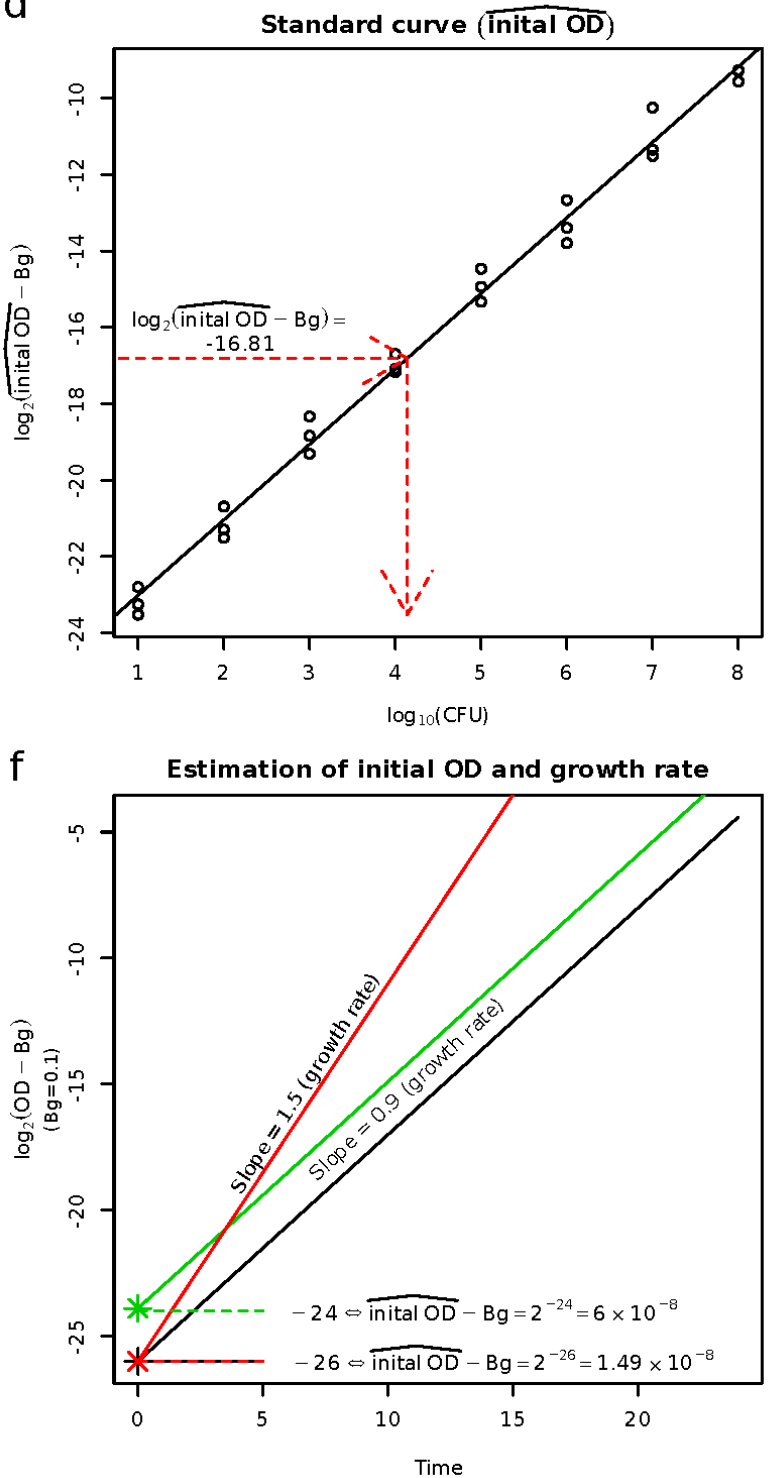

Figure A1: Estimation of the \#EqNC by both the Start Growth Time (SGT; [50]) and initial OD (our approach).

Left and right panels respectively refer to the SGT and initial OD approaches. Panels a and b: representative real data as analyzed by each method, raw data for SGT and log of the 
OD minus the background (Bg) for initial OD (see Supplementary method 1 for background estimation). Panel a: the SGT approach yields an estimated SGT of 13, using a threshold of 0.105. Panel b: the linear model gives initial OD-Bg $=2^{-16.81}$. Panels $\mathrm{c}$ and $\mathrm{d}$ : The standard curve is obtained by quantifying cells by both counting CFUs and analyzing the growth curves whatever the approach (SGT or initial OD). If growth rates were similar when computing and using the standard curve, both approaches gave similar results. However, as illustrated in panels e and f, the SGT approach started to be inaccurate when growth rates varied. Panels e and f: Simulated examples showing both the inaccuracy of the SGT approach and the robustness of the initial OD approach in the presence of growth rate variation. Panel e: three theoretical growth curves obtained by assuming growth rates of 1.5, 0.9 , and 0.9 cell division per hour, for initial ODs of $2^{-26}, 2^{-24}$, and 2-26, respectively for the red, green and black curves. The SGT approach based on the OD threshold would detect more cells in, successively, the red, green and black curves. However, as illustrated in the inset zoom, this is an artefact induced by growth rate variation. Panel f: log-transformed OD allows the fit of a linear model that yields an estimate of both the growth rate and initial OD $\left(\log _{2}(\right.$ initial OD-Bg) $)$.

\section{References}

1. Interagency Coordination Group on Antimicrobial Resistance No Time to Wait: Securing the Future from DrugResistant Infections 2019.

2. Kraker, M.E.A.D.; Stewardson, A.J.; Harbarth, S. Will 10 Million People Die a Year Due to Antimicrobial Resistance by 2050 ? PLoS Medecine 2016, 1-6, doi:10.1371/journal.pmed.1002184.

3. Cassini, A.; Högberg, L.D.; Plachouras, D.; Quattrocchi, A.; Hoxha, A.; Simonsen, G.S.; Colomb-Cotinat, M.; Kretzschmar, M.E.; Devleesschauwer, B.; Cecchini, M.; et al. Attributable Deaths and Disability-Adjusted Life-Years Caused by Infections with Antibiotic-Resistant Bacteria in the EU and the European Economic Area in 2015: A Population-Level Modelling Analysis. The Lancet infectious diseases 2019, 19, 56-66.

4. Brauner, A.; Fridman, O.; Gefen, O.; Balaban, N.Q. Distinguishing between Resistance, Tolerance and Persistence to Antibiotic Treatment. Nat Rev Microbiol 2016, 14, 320-330, doi:10.1038/nrmicro.2016.34.

5. Balaban, N.Q.; Helaine, S.; Lewis, K.; Ackermann, M.; Aldridge, B.; Andersson, D.I.; Brynildsen, M.P.; Bumann, D.; Camilli, A.; Collins, J.J.; et al. Definitions and Guidelines for Research on Antibiotic Persistence. Nat Rev Microbiol 2019, 17, 441-448, doi:10.1038/s41579-019-0196-3.

6. Bakkeren, E.; Diard, M.; Hardt, W.-D. Evolutionary Causes and Consequences of Bacterial Antibiotic Persistence. Nat Rev Microbiol 2020, 18, 479-490, doi:10.1038/s41579-020-0378-z.

7. Van den Bergh, B.; Fauvart, M.; Michiels, J. Formation, Physiology, Ecology, Evolution and Clinical Importance of Bacterial Persisters. FEMS Microbiology Reviews 2017, 41, 219-251, doi:10.1093/femsre/fux001.

8. Jung, S.-H.; Ryu, C.-M.; Kim, J.-S. Bacterial Persistence: Fundamentals and Clinical Importance. J Microbiol. 2019, 57, 829-835, doi:10.1007/s12275-019-9218-0.

9. Cohen, N.R.; Lobritz, M.A.; Collins, J.J. Microbial Persistence and the Road to Drug Resistance. Cell Host and Microbe 2013, 13, 632-642, doi:10.1016/j.chom.2013.05.009.

10. Sharma, S.V.; Lee, D.Y.; Li, B.; Quinlan, M.P.; Takahashi, F.; Maheswaran, S.; McDermott, U.; Azizian, N.; Zou, L.; Fischbach, M.A.; et al. A Chromatin-Mediated Reversible Drug-Tolerant State in Cancer Cell Subpopulations. Cell 2010, 141, 69-80, doi:10.1016/j.cell.2010.02.027.

11. Swayden, M.; Chhouri, H.; Anouar, Y.; Grumolato, L. Tolerant/Persister Cancer Cells and the Path to Resistance to Targeted Therapy. Cells 2020, 9, 2601, doi:10.3390/cells9122601. 
12. Vallette, F.M.; Olivier, C.; Lézot, F.; Oliver, L.; Cochonneau, D.; Lalier, L.; Cartron, P.-F.; Heymann, D. Dormant, Quiescent, Tolerant and Persister Cells: Four Synonyms for the Same Target in Cancer. Biochemical Pharmacology 2019, 162, 169-176, doi:10.1016/j.bcp.2018.11.004.

13. Lehman, J.; Clune, J.; Misevic, D.; Adami, C.; Altenberg, L.; Beaulieu, J.; Bentley, P.J.; Bernard, S.; Beslon, G.; Bryson, D.M.; et al. The Surprising Creativity of Digital Evolution: A Collection of Anecdotes from the Evolutionary Computation and Artificial Life Research Communities. Artificial Life 2020, 26, 274-306, doi:10.1162/artl_a_00319.

14. Fisher, R.A.; Gollan, B.; Helaine, S. Persistent Bacterial Infections and Persister Cells. Nat Rev Microbiol 2017, 15, 453-464, doi:10.1038/nrmicro.2017.42.

15. Gollan, B.; Grabe, G.; Michaux, C.; Helaine, S. Bacterial Persisters and Infection: Past, Present, and Progressing. Annu. Rev. Microbiol. 2019, 73, 359-385, doi:10.1146/annurev-micro-020518-115650.

16. Fauvart, M.; De Groote, V.N.; Michiels, J. Role of Persister Cells in Chronic Infections: Clinical Relevance and Perspectives on Anti-Persister Therapies. Journal of Medical Microbiology 2011, 60, 699-709, doi:10.1099/jmm.0.030932-0.

17. Li, L.; Mendis, N.; Trigui, H.; Oliver, J.D.; Faucher, S.P. The Importance of the Viable but Non-Culturable State in Human Bacterial Pathogens. Front. Microbiol. 2014, 5, doi:10.3389/fmicb.2014.00258.

18. Lin, P.L.; Flynn, J.L. Understanding Latent Tuberculosis: A Moving Target. J.I. 2010, 185, 15-22, doi:10.4049/jimmunol.0903856.

19. Windels, E.M.; Michiels, J.E.; Fauvart, M.; Wenseleers, T.; Van den Bergh, B.; Michiels, J. Bacterial Persistence Promotes the Evolution of Antibiotic Resistance by Increasing Survival and Mutation Rates. ISME J 2019, 13, 1239 1251, doi:10.1038/s41396-019-0344-9.

20. Bigger, J. Treatment of Staphyloeoeeal Infections with Penicillin by Intermittent Sterilisation. Lancet 1944, 497-500.

21. Balaban, N.Q.; Merrin, J.; Chait, R.; Kowalik, L.; Leibler, S. Bacterial Persistence as a Phenotypic Switch. Science 2004, $305,5$.

22. Suter, L.; Widmer, A. Phenotypic Effects of Salt and Heat Stress over Three Generations in Arabidopsis Thaliana. PLoS ONE 2013, 8, e80819, doi:10.1371/journal.pone.0080819.

23. Badyaev, A.V. Environmental Stress and Developmental Stability in Dentition of the Yellowstone Grizzly Bears. Behavioral Ecology 1998, 9, 339-344, doi:10.1093/beheco/9.4.339.

24. Vøllestad, L.A.; Hindar, K. Developmental Stability and Environmental Stress in Salmo Salar (Atlantic Salmon). Heredity 1997, 78, 215-222.

25. Hurtado, L.; Castrezana, S.; Mateos, M.; Mclaurin, D.; Tello, M.K.; Campoy, J.; Markow, T. Developmental Stability and Environmental Stress in Natural Populations of Drosophila Pachea. Ecotoxicology 1997, 6, 233-238.

26. Badyaev, A.V. Stress-Induced Variation in Evolution: From Behavioural Plasticity to Genetic Assimilation. Proceedings. Biological sciences / The Royal Society 2005, 272, 877-886, doi:10.1098/rspb.2004.3045.

27. Vogt, G. Disentangling the Environmentally Induced and Stochastic Developmental Components of Phenotypic Variation. In Phenotypic switching: implications in biology and medicine; Elsevier, 2020; pp. 207-251 ISBN 978-0-12817996-3.

28. Vogt, G. Stochastic Developmental Variation, an Epigenetic Source of Phenotypic Diversity with Far-Reaching Biological Consequences. J Biosci 2015, 40, 159-204, doi:10.1007/s12038-015-9506-8.

29. Krishna, S.; Laxman, S. Emergence of Metabolic Heterogeneity in Cell Populations: Lessons from Budding Yeast. In Phenotypic switching: implications in biology and medicine; Elsevier, 2020; pp. 335-360 ISBN 978-0-12-817996-3. 
30. Schwartz, M.H.; Waldbauer, J.R.; Zhang, L.; Pan, T. Global TRNA Misacylation Induced by Anaerobiosis and Antibiotic Exposure Broadly Increases Stress Resistance in Escherichia Coli. Nucleic Acids Res 2016, gkw856, doi:10.1093/nar/gkw856.

31. Rocabert, C.; Beslon, G.; Knibbe, C.; Bernard, S. Phenotypic Noise and the Cost of Complexity; Evolutionary Biology, 2020;

32. Wang, Q.; Huang, L.; Wen, K.; Yu, J.; ,Center for Applied Mathematics, Guangzhou University, Guangzhou 510006, China; ,College of Science, Guangxi University of Science and Technology, Liuzhou 545006, China; ,School of Statistics and Mathematics, Guangdong University of Finance and Economics, Guangzhou 510275, China The Mean and Noise of Stochastic Gene Transcription with Cell Division. Mathematical Biosciences E Engineering 2018, 15, 12551270, doi:10.3934/mbe.2018058.

33. Prado Casanova, M. Noise and Synthetic Biology: How to Deal with Stochasticity? Nanoethics 2020, 14, 113-122, doi:10.1007/s11569-020-00366-4.

34. Kaneko, K.; Furusawa, C. Relevance of Phenotypic Noise to Adaptation and Evolution. IET Systems Biology 2008, 2, 234-246, doi:10.1049/iet-syb:20070078.

35. Hallgrimsson, B.; Willmore, K.; Hall, B.K. Canalization, Developmental Stability, and Morphological Integration in Primate Limbs. Am. J. Phys. Anthropol. 2002, 119, 131-158, doi:10.1002/ajpa.10182.

36. Ortbauer, M. Abiotic Stress Adaptation: Protein Folding Stability and Dynamics. In Abiotic Stress - Plant Responses and Applications in Agriculture; Vahdati, K., Ed.; InTech, 2013 ISBN 978-953-51-1024-8.

37. Jorgen, R.; Helen, O.; Niclas, J. What Is Bet-Hedging, Really? Proceedings of the Royal Society B 2010, 277, 1153-1154, doi:10.1086/284396.

38. Levin, B.R.; Concepción-Acevedo, J.; Udekwu, K.I. Persistence: A Copacetic and Parsimonious Hypothesis for the Existence of Non-Inherited Resistance to Antibiotics. Current Opinion in Microbiology 2014, 21, 18-21, doi:10.1016/j.mib.2014.06.016.

39. Girgis, H.S.; Harris, K.; Tavazoie, S. Large Mutational Target Size for Rapid Emergence of Bacterial Persistence. Proceedings of the National Academy of Sciences 2012, 109, 12740-12745, doi:10.1073/pnas.1205124109.

40. Nichol, D.; Robertson, M.; Jeavons, P.; Anderson, A.R.A. Stochasticity in the Genotype-Phenotype Map: Implications for the Robustness and Persistence of Bet-Hedging. Genetics 2016, 204, 1523-1539.

41. Pu, Y.; Ke, Y.; Bai, F. Active Efflux in Dormant Bacterial Cells - New Insights into Antibiotic Persistence. Drug Resistance Updates 2017, 30, 7-14, doi:10.1016/j.drup.2016.11.002.

42. Pu, Y.; Zhao, Z.; Li, Y.; Zou, J.; Ma, Q.; Zhao, Y.; Ke, Y.; Zhu, Y.; Chen, H.; Baker, M.A.B.; et al. Enhanced Efflux Activity Facilitates Drug Tolerance in Dormant Bacterial Cells. Molecular Cell 2016, 62, 284-294, doi:10.1016/j.molcel.2016.03.035.

43. LaFleur, M.D.; Qi, Q.; Lewis, K. Patients with Long-Term Oral Carriage Harbor High-Persister Mutants of Candida Albicans. AAC 2010, 54, 39-44, doi:10.1128/AAC.00860-09.

44. Stepanyan, K.; Wenseleers, T.; Duéñez-Guzmán, E.A.; Muratori, F.; Van den Bergh, B.; Verstraeten, N.; De Meester, L.; Verstrepen, K.J.; Fauvart, M.; Michiels, J. Fitness Trade-Offs Explain Low Levels of Persister Cells in the Opportunistic Pathogen Pseudomonas Aeruginosa. Mol Ecol 2015, 24, 1572-1583, doi:10.1111/mec.13127.

45. Goneau, L.W.; Yeoh, N.S.; MacDonald, K.W.; Cadieux, P.A.; Burton, J.P.; Razvi, H.; Reid, G. Selective Target Inactivation Rather than Global Metabolic Dormancy Causes Antibiotic Tolerance in Uropathogens. Antimicrob. Agents Chemother. 2014, 58, 2089-2097, doi:10.1128/AAC.02552-13. 
46. Vulin, C.; Leimer, N.; Huemer, M.; Ackermann, M.; Zinkernagel, A.S. Prolonged Bacterial Lag Time Results in Small Colony Variants That Represent a Sub-Population of Persisters. Nat Commun 2018, 9, 4074, doi:10.1038/s41467-01806527-0.

47. Van den Bergh, B.; Michiels, J.E.; Wenseleers, T.; Windels, E.M.; Boer, P.V.; Kestemont, D.; De Meester, L.; Verstrepen, K.J.; Verstraeten, N.; Fauvart, M.; et al. Frequency of Antibiotic Application Drives Rapid Evolutionary Adaptation of Escherichia Coli Persistence. Nat Microbiol 2016, 1, 16020, doi:10.1038/nmicrobiol.2016.20.

48. Good, B.H.; Mcdonald, M.J.; Barrick, J.E.; Lenski, R.E.; Desai, M.M. The Dynamics of Molecular Evolution over 60,000 Generations. Nature 2017, 551, 45-50, doi:10.1038/nature24287.

49. Lamrabet, O.; Martin, M.; Lenski, R.E.; Schneider, D. Changes in Intrinsic Antibiotic Susceptibility during a LongTerm Evolution Experiment with Escherichia Coli. mBio 2019, 10, e00189-19, /mbio/10/2/mBio.00189-19.atom, doi:10.1128/mBio.00189-19.

50. Hazan, R.; Que, Y.-A.; Maura, D.; Rahme, L.G. A Method for High Throughput Determination of Viable Bacteria Cell Counts in 96-Well Plates. BMC Microbiol 2012, 12, 259, doi:10.1186/1471-2180-12-259.

51. Plucain, J.; Hindre, T.; Le Gac, M.; Tenaillon, O.; Cruveiller, S.; Medigue, C.; Leiby, N.; Harcombe, W.R.; Marx, C.J.; Lenski, R.E.; et al. Epistasis and Allele Specificity in the Emergence of a Stable Polymorphism in Escherichia Coli. Science 2014, 343, 1366-1369, doi:10.1126/science.1248688.

52. Wiser, M.J.; Ribeck, N.; Lenski, R.E. Long-Term Dynamics of Adaptation in Asexual Populations. Science 2013, 342, 1364-1367.

53. Lenski, R.E.; Rose, M.R.; Simpson, S.C.; Tadler, S.C. Long-Term Experimental Evolution in Escherichia Coli. I. Adaptation and Divergence During 2,000 Generations. The American Naturalist 1991, 138, 1315-1341.

54. Consuegra, J.; Plucain, J.; Gaffé, J.; Hindré, T.; Schneider, D. Genetic Basis of Exploiting Ecological Opportunity During the Long-Term Diversification of a Bacterial Population. J Mol Evol 2017, 85, 26-36, doi:10.1007/s00239-0179802-z.

55. Vogwill, T.; Comfort, A.C.; Furió, V.; MacLean, R.C. Persistence and Resistance as Complementary Bacterial Adaptations to Antibiotics. J. Evol. Biol. 2016, 29, 1223-1233, doi:10.1111/jeb.12864.

56. Hofsteenge, N.; van Nimwegen, E.; Silander, O.K. Quantitative Analysis of Persister Fractions Suggests Different Mechanisms of Formation among Environmental Isolates of E. Coli. BMC Microbiol 2013, 13, 25, doi:10.1186/14712180-13-25.

57. Stewart, B.; Rozen, D.E. Genetic Variation for Antibiotic Persistence in Escherichia Coli. Evolution 2012, 66, 933-939, doi:10.1111/j.1558-5646.2011.01467.x.

58. Roemhild, R.; Gokhale, C.S.; Dirksen, P.; Blake, C.; Rosenstiel, P.; Traulsen, A.; Andersson, D.I.; Schulenburg, H. Cellular Hysteresis as a Principle to Maximize the Efficacy of Antibiotic Therapy. Proc Natl Acad Sci USA 2018, 115, 9767-9772, doi:10.1073/pnas.1810004115.

59. Roemhild, R.; Schulenburg, H. Evolutionary Ecology Meets the Antibiotic Crisis. Evolution, Medicine, and Public Health 2019, 2019, 37-45, doi:10.1093/emph/eoz008.

60. Mitchell, A.; Romano, G.H.; Groisman, B.; Yona, A.; Dekel, E.; Kupiec, M.; Dahan, O.; Pilpel, Y. Adaptive Prediction of Environmental Changes by Microorganisms. Nature 2009, 460, 220-224, doi:10.1038/nature08112.

61. López García de Lomana, A.; Kaur, A.; Turkarslan, S.; Beer, K.D.; Mast, F.D.; Smith, J.J.; Aitchison, J.D.; Baliga, N.S. Adaptive Prediction Emerges over Short Evolutionary Time Scales. Genome Biology and Evolution 2017, 9, 1616-1623, doi:10.1093/gbe/evx116.

62. Rozen, D.E.; Lenski, R.E. Long-Term Experimental Evolution in Escherichia Coli. VIII. Dynamics of a Balanced Polymorphism. 2000, 12. 
63. Rozen, D.E.; Philippe, N.; Arjan de Visser, J.; Lenski, R.E.; Schneider, D. Death and Cannibalism in a Seasonal Environment Facilitate Bacterial Coexistence. Ecology Letters 2009, 12, 34-44, doi:10.1111/j.1461-0248.2008.01257.x.

64. Großkopf, T.; Consuegra, J.; Gaffé, J.; Willison, J.C.; Lenski, R.E.; Soyer, O.S.; Schneider, D. Metabolic Modelling in a Dynamic Evolutionary Framework Predicts Adaptive Diversification of Bacteria in a Long-Term Evolution Experiment. BMC Evol Biol 2016, 16, 163, doi:10.1186/s12862-016-0733-x.

65. Keren, I.; Kaldalu, N.; Spoering, A.; Wang, Y.; Lewis, K. Persister Cells and Tolerance to Antimicrobials. FEMS Microbiology Letters 2004, 230, 13-18, doi:10.1016/S0378-1097(03)00856-5.

66. Jeong, H.; Barbe, V.; Lee, C.H.; Vallenet, D.; Yu, D.S.; Choi, S.-H.; Couloux, A.; Lee, S.-W.; Yoon, S.H.; Cattolico, L.; et al. Genome Sequences of Escherichia Coli B Strains REL606 and BL21(DE3). Journal of Molecular Biology 2009, 394, 644-652, doi:10.1016/j.jmb.2009.09.052.

67. Rozen, D.E.; Schneider, D.; Lenski, R.E. Long-Term Experimental Evolution in Escherichia Coli. XIII. Phylogenetic History of a Balanced Polymorphism. J Mol Evol 2005, 61, 171-180, doi:10.1007/s00239-004-0322-2.

68. Bates, D.; Mächler, M.; Bolker, B.; Walker, S. Fitting Linear Mixed-Effects Models Using Lme4. Journal of Statistical Software 2015, 67, 1-48, doi:10.18637/jss.v067.i01.

69. Luke, S.G. Evaluating Significance in Linear Mixed-Effects Models in R. Behav Res 2017, 49, 1494-1502, doi:10.3758/s13428-016-0809-y.

70. Li, P.; Redden, D.T. Comparing Denominator Degrees of Freedom Approximations for the Generalized Linear Mixed Model in Analyzing Binary Outcome in Small Sample Cluster-Randomized Trials. BMC Med Res Methodol 2015, 15, 38, doi:10.1186/s12874-015-0026-x.

71. Hothorn, T.; Bretz, F.; Westfall, P. Simultaneous Inference in General Parametric Models. Biometrical Journal 2008, 50, 346-363, doi:10.1002/bimj.200810425. 


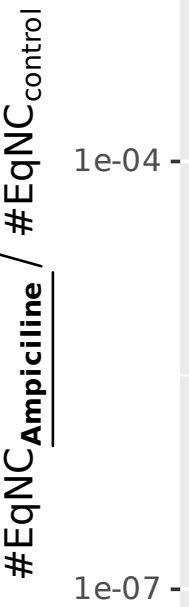

- Mutator

- Non-mutator 


$$
\begin{gathered}
\text { Ara- } 1 \\
x=1 ; \Delta=1
\end{gathered}
$$

$$
\begin{gathered}
\text { Ara }+1 \\
\times=1 ; \boldsymbol{\Delta}=1
\end{gathered}
$$$$
x=1 ; \Delta=0.22
$$

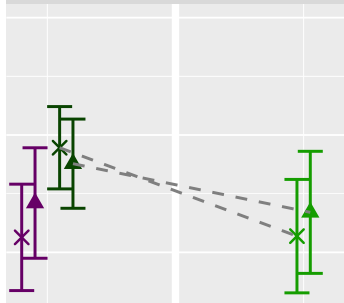
$1 e-06$ -

1e-02 -
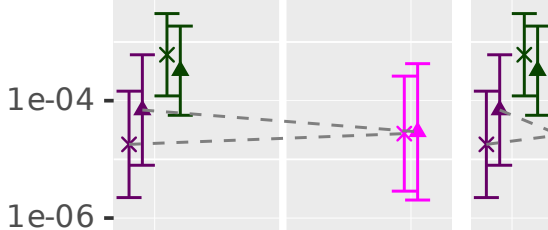

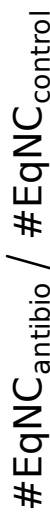

\section{Ara-2}

$\mathrm{L}: \mathrm{x}=1 ; \boldsymbol{\Delta}=1$

S: $x=1 ; \boldsymbol{\Delta}=0.015 *$

$$
\begin{gathered}
\text { Ara-5 } \\
\times=1 ; \Delta=1
\end{gathered}
$$

$1 e-02$

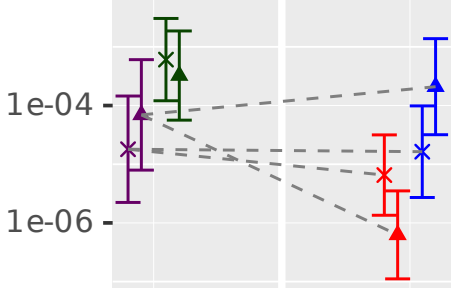

$$
\begin{gathered}
\text { Ara-3 } \\
x=1 ; \Delta=1
\end{gathered}
$$

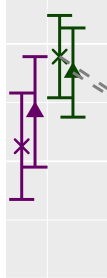

$$
\begin{gathered}
\text { Ara }+3 \\
x=1 ; \boldsymbol{\Delta}=1
\end{gathered}
$$

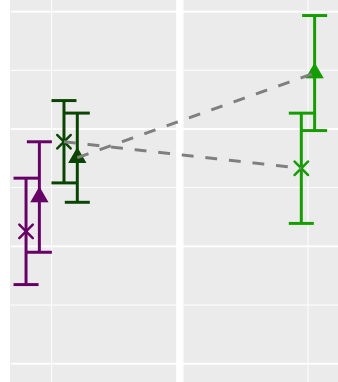

$$
\text { Ara }+4
$$

$$
x=1 ; \Delta=0.49
$$

$$
\begin{gathered}
\text { Ara }+5 \\
\times=1 ; \boldsymbol{\Delta}=1
\end{gathered}
$$

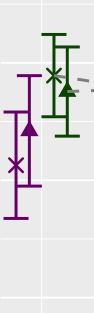

$$
\begin{gathered}
\text { Ara }+6 \\
\times=1 ; \boldsymbol{\Delta}=1
\end{gathered}
$$

1e-02 -
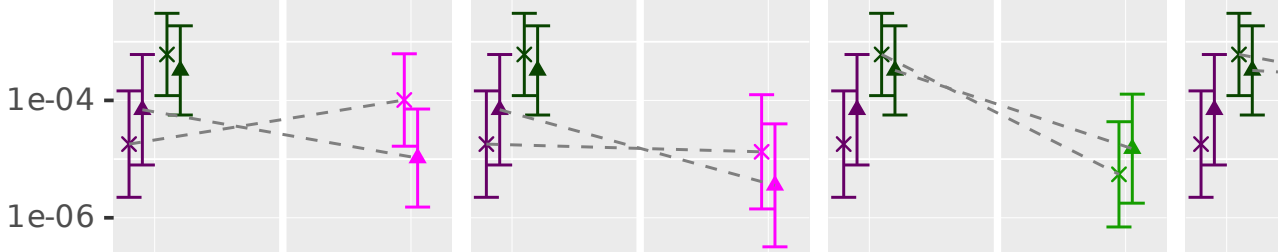

ó 50000 ó 


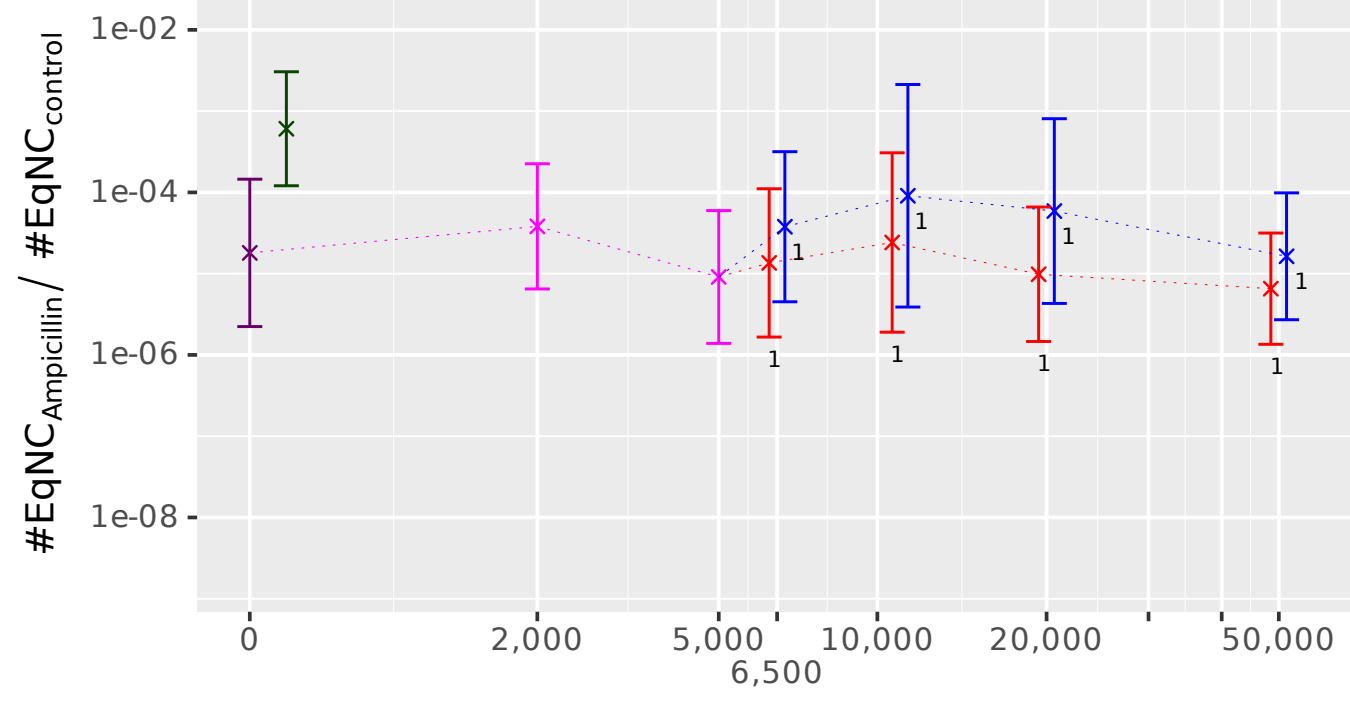

\section{Generation}

\section{B Ciprofloxacin}

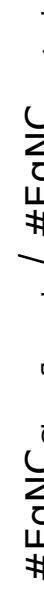
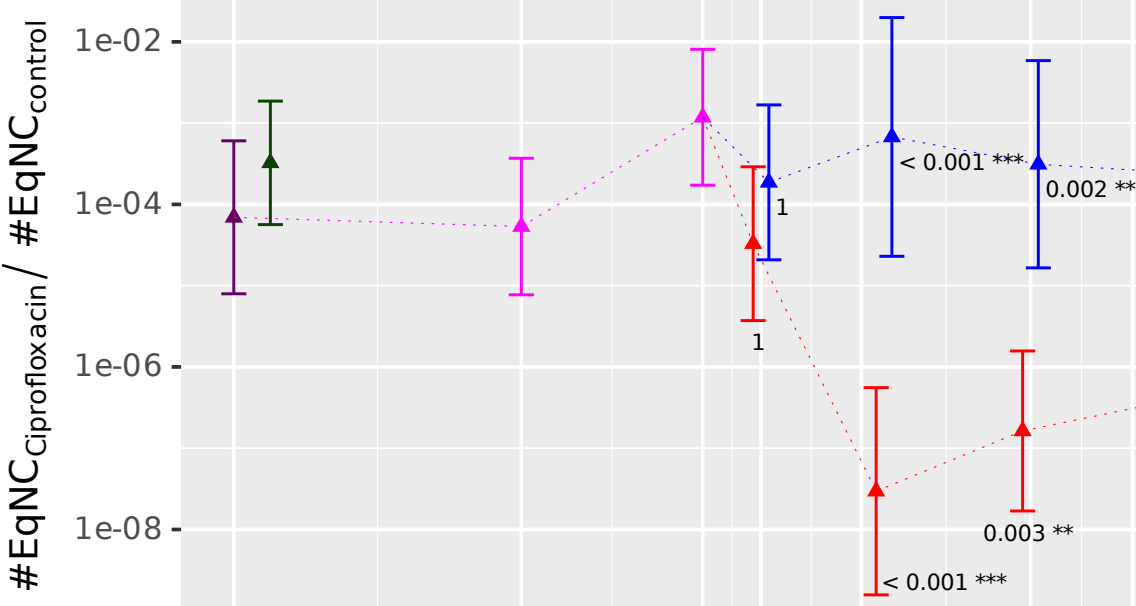

$\dot{0} \quad 2,000$

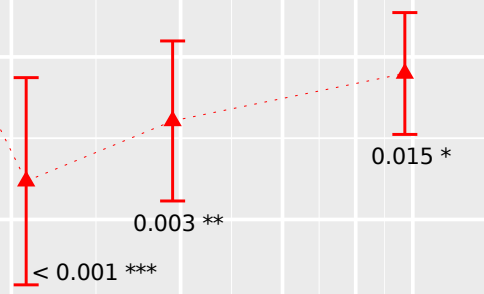
$5,0000^{\prime} \quad 10,000 \quad 20,000 \quad ' \quad 50,000$ 6,500

Generation 